\title{
Focus on antimicrobial use in the era of increasing antimicrobial resistance in ICU
}

\author{
Matteo Bassetti ${ }^{1}$, Garyphallia Poulakou ${ }^{2}$ and Jean-François Timsit ${ }^{3,4^{*}}$
}

(c) 2016 Springer-Verlag Berlin Heidelberg and ESICM

\section{Antibiotic stewardship in the spectrum of resistance bacteria fight}

Antimicrobial resistance in microorganisms increased inexorably driven by antimicrobial exposure in healthcare, agriculture, and the environment. Onward transmission is affected by standards of infection control, sanitation, access to clean water, access to quality-assured antimicrobials and diagnostic tests, travel, and migration $[1,2]$. The intensive care unit (ICU) represents the best bacterial resistance amplifier. Indeed, the most critically ill patients with invasive procedures are treated with broad-spectrum antimicrobials in an environment with a huge number of healthcare workers and an extremely high risk of transmission from patient to patient.

Strategies to reduce curative antibiotic therapy to the bare essential are therefore needed and should include an immediate diagnostic process before starting early probabilistic antimicrobial therapy in case of severe sepsis or septic shock. The strategies proposed by a French multidisciplinary panel of experts using GRADE methods are detailed in this journal (67 recommendations) [3]. The key messages are that all antibiotic usage promotes antibiotic resistance. Adequate high dose is essential to cure patients but treatment should spare carbapenems in community-acquired infections. Combination therapy is suggested for patients with septic shock and neutropenia or in patients at high risk of multidrug-resistant bacterias. Antibiotics should be reassessed after $48-72 \mathrm{~h}$ and deescalated. Duration of therapy should be reduced. In contrast, in the absence of severe sepsis, waiting for objective data to diagnose infection before treatment with antimicrobial drugs for suspected ICU-acquired infections does

\footnotetext{
*Correspondence: jean-francois.timsit@bch.aphp.fr

${ }^{3}$ Université Paris Diderot/Hopital Bichat, Réanimation Medicale et des maladies infectieuses, Paris, France

Full author information is available at the end of the article
}

not worsen mortality and might be associated with better outcomes and use of antimicrobial drugs and should be discussed on daily rounds [4].

Among Gram-negative bacteria, Acinetobacter baumannii is particularly equipped to spread in ICUs and to become multiresistant. It became the first agent responsible for nosocomial infection in ICUs in many countries. Infection control strategies including hand hygiene, environmental cleaning, active screening, and contact precautions should therefore be added to antimicrobial stewardship programs. A task force organized by the infection section of the European Society of Intensive Care Medicine (ESICM) detailed strategies to control A. baumannii spread and adequate treatment regimens that should be used in case of $A$. baumannii infections [5].

In order to reduce cross transmission of bacteria, hand hygiene might not be sufficient because of imperfect compliance. An alternative approach may focus on limiting the reservoir of potential pathogens on the skin and in the mouth and gut of the patients. Whereas $2 \%$ alcoholic chlorhexidine became the first-choice antiseptic for catheter infection prevention [6], the use of chlorhexidine as a topical agent for decolonization of a patient's mouth and skin is still debated. The recent review by Noto and Wheeler [7] suggests that cutaneous decolonization with chlorhexidine results in less Gram-positive multidrugresistant acquisition (methicillin-resistant Staphylococcus aureus (MRSA) and vancomycin-resistant enterococci (VRE)). The impact on bacteremia and nosocomial infections is largely driven by a decrease of coagulase-negative bacteremia, and available studies do not demonstrate any effect on Gram-negative infections. The benefit of chlorhexidine-based oropharyngeal decolonization is small and also requires further confirmation. Chlorhexidine use was also associated with the rise of infections due to organisms with reduced susceptibility and with rare occurrence of allergic or toxic reactions.

\section{Springer}


Therefore, Noto and Wheeler concluded that the chlorhexidine-based decolonization should not be used widely. It should therefore remain in the armamentarium of strategies to prevent the spread of multidrug-resistant (MDR) microorganisms in case of epidemic.

\section{Empiric treatment}

Previous studies have shown conflicting evidence regarding the impact of inappropriate, initial antibiotic therapy in septic patients, but in the case of patients with severe sepsis or septic shock, survival depends on early initiation of effective antimicrobial treatment [8]. The choice of empiric appropriate therapy, meaning appropriate and early therapy at pharmacokinetic/pharmacodynamic (PK/PD)-optimized doses and dose intervals, is currently complex. MDR microorganisms are increasing, and inappropriate empirical treatment has been described as an independent predictor of 14-day mortality (OR, 1.48; $95 \%$ CI, 1.01-2.18) for infections caused by Klebsiella pneumoniae (Kp)-producing carbapenemase (KPC) [9]. Moreover, in critically ill patients physiological alterations may severely modify antibiotic exposure. A post hoc analysis of the DALI study, simulating an empirical setting, found that target attainment during therapy with beta-lactam (BL) antibiotics was overall inadequate. Besides, use of intermittent bolus (IB) infusion resulted in a 3- to 4 -fold increase in the likelihood of not reaching the desired PK/PD targets [10]. Nevertheless, the most recent relevant multicenter prospective clinical studies regarding $\mathrm{PK} / \mathrm{PD}$ targets for BL antibiotics in critically ill patients have reached contradictory conclusions. The BLISS study observed that in patients with severe sepsis (without renal replacement), continuous infusion (CI) administration was associated with higher clinical cure rates (56 versus $34 \%, p=0.011$ ) and better $\mathrm{PK} / \mathrm{PD}$ target attainment compared to IB dosing for BL antibiotics [11]. In contrast, the BLING II study did not observe difference in clinical cure (52.4 versus $49.5 \%$, $p=0.56)$ between $\mathrm{BL}$ antibiotic administration by CI and IB [12]. These differences between the results may be explained by epidemiological settings, since in the second trial, the prevalence of MDR pathogens was very low and the probability of not reaching the $\mathrm{PK} / \mathrm{PD}$ target using conventional dosing was very small. Furthermore, patients with acute renal failure, for whom continuous infusion could not improve PK, were included in BLING II and excluded in BLISS.

In addition, individual or local nosocomial risk factors, including colonization status with MDR bacteria, should support empirical treatment decisions [8].

\section{Adequate dosing}

The DALI study, a prospective, multinational pharmacokinetic point-prevalence study, was a breakthrough in our knowledge on antibiotic dosing in ICU patients [13]. Significant rates of insufficient $\beta$-lactam exposure were observed, with approximately $20 \%$ of patients failing to attain the most conservative drug exposure targets $(50 \%$ $f T_{>\text {MIC }}$, where $f T_{>\text {MIC }}$ is the percentage of a 24-h time period that the unbound drug concentration exceeds the minimum inhibitory concentration, MIC) during empirical treatment, whereas more than $40 \%$ failed to achieve a higher target $\left(100 \% f T_{>\text {MIC }}\right)$. Intermittent infusion of $\beta$-lactams and patients with augmented renal clearance were associated with lower drug exposures [10]. In line with these results was a randomized study by de Waele et al. in ICU patients with normal renal function, reporting very low baseline PK/PD target attainment both for those receiving piperacillin/tazobactam and meropenem. Among those randomized to daily therapeutic drug monitoring (TDM) and adaptation of doses, a higher proportion of patients attained both the $100 \% f T_{>\mathrm{MIC}}$ and $100 \%$ $f T_{>4 \mathrm{MIC}}$ target [14]. Apart from disease severity, increasing rates of PD target attainment were associated with better clinical outcomes [13]. Continuous infusion of $\beta$-lactams was associated with better clinical outcomes in a recent randomized controlled trial [12]. In light of these data, current empiric dosing recommendations of $\beta$-lactam antibiotics for ICU patients seem inadequate and need to be reconsidered [10].

Close monitoring of amikacin concentrations seems mandatory in ICU patients; despite a loading dose of $25 \mathrm{mg} / \mathrm{kg}$ of total body weight, the PD target was not attained in $33 \%$ of patients. Positive 24-h fluid balance was predictive of target non-attainment, whereas low BMI tended to be associated with amikacin underdosing [15].

Usage of tigecycline was explored in a prospective observational study from 26 French ICUs; $65 \%$ of patients received a combination with other antibiotics mostly targeted against Gram-negative infections and $94 \%$ received the standard dose. Success rates of $65 \%$ for patients alive at the end of treatment were comparable to clinical studies of severe infections, tending to decrease with illness severity, immunosuppression, bacteremia, and obesity [16]. Given that most patients received combination therapy, the study did not add much to the ongoing discussion of whether the current recommended scheme of $50 \mathrm{mg}$ BID is adequate or not. However, the task force on management and prevention of A. baumannii infections in the ICU organized by the infection section of ESICM recommended a loading dose of $200 \mathrm{mg}$ of tigecycline followed by $100 \mathrm{mg}$ BID for the treatment of pneumonia and primary bacteremia [5]. 
Linezolid's plasma and pulmonary concentrations have been questioned in critically ill and obese patients. A randomized controlled study by De Pascale et al. comparing intermittent vs continuous infusion in moderately obese patients with VAP demonstrated suboptimal plasma concentrations with intermittent infusion. Continuous infusion overcame this issue, except for extreme MIC values above $4 \mathrm{mg} / \mathrm{L}$ and was associated with higher alveolar penetration and epithelial lining fluid (ELF) concentrations above $4 \mathrm{mg} / \mathrm{L}$ [17].

\section{Consider de-escalation}

De-escalation of antimicrobial therapy is a strategy to reduce the spectrum of antimicrobials and aims to prevent the emergence of bacterial resistance [18]. The quality of the studies analyzing the effect of de-escalation is weak. There is no uniform definition of de-escalation that includes the decrease of the number of antimicrobials, the shortening of the duration of antibiotic therapy, and the switch of the pivotal antibiotic to a narrower agent. Available studies suggested an association between deescalation and a better outcome.

Garnacho-Montero et al. designed a prospective observational cohort of 712 patients with severe sepsis. De-escalation was defined as a discontinuation of an antimicrobial agent or change of antibiotic to one with the narrowest spectrum [19]. They found that de-escalation was only applied in $35 \%$ of the cases and was associated with a significantly lower risk of death even after adjustment based on risk factors of death. In contrast, in an open randomized non-inferiority trial, Leone et al. [20] failed to demonstrate the non-inferiority of narrowing the spectrum of the pivotal antimicrobial on the duration of ICU stay. The intervention also increased the rates of superinfection and was paradoxically associated with more days on antibiotic. This paradoxical effect of de-escalation is partly due to an unexpected disequilibrium between both groups in terms of source of infection and initial use of carbapenems and fluoroquinolones. The decrease of antibiotic pressure to reduce antibiotic resistance is therefore challenging and may need optimization of the pharmacokinetics of the antimicrobials given [10] and a better understanding of the impact of antimicrobials on the human, mainly gut, microbiota.

The applicability of de-escalation in MDR infections has not been extensively explored and should be considered individually. In this setting de-escalation implies mostly reducing the antibiotic treatment duration on the basis of the patient's clinical response and the underlying infection. To help in shortening the duration of antibiotic therapy the most promising parameter appears to be plasma levels of procalcitonin [21]. In addition, development of rapid diagnostic tools and antimicrobial susceptibility testing are needed.

Table 1 summarizes the principal components of ICU strategies for stewardship and antimicrobial use.

\section{Table 1 Principal components of ICU strategies for stewardship and antimicrobial use in the era of increasing antimicro-} bial resistance

\begin{tabular}{l} 
Leadership commitment \\
Implementing antibiotic stewardship programs \\
Implementing infection control practices \\
Improve communication between laboratory and clinical staff \\
Implement local resistance data for developing local antibiotic guidelines \\
Multidisciplinary approach \\
A multidisciplinary team including infectious diseases specialists, microbiologists, pharmacists, ICU physicians, and nurses should be in charge of \\
developing a specific ICU antibiotic stewardship program \\
Daily rounds for case discussions \\
Implementation of modern antimicrobial use approach \\
Aggressive good quality microbiological sampling (blood cultures; distal airway sampling; urine culture; systematic sampling of wound, drain dis- \\
charge, and any collection suspected of being infected) before starting new antimicrobials \\
Selection of empirical antimicrobials according to the clinical conditions, the presence of risk factors for resistant microorganisms, and the local epide- \\
miology \\
Achievement of adequate pharmacokinetic/pharmacodynamic parameters of the antimicrobial agents used (extended/continuous infusion, TDM) \\
Systematic de-escalation \\
Systematic reduction of the duration of antimicrobial treatment according to the clinical evolution and the kinetics of biomarkers such as procalci- \\
tonin \\
Monitoring and feedback \\
Monitoring antibiotic prescribing and resistance patterns \\
Regular reporting of information on antibiotic use and resistance to doctors, nurses, and relevant staff \\
\hline
\end{tabular}




\section{Abbreviations}

BL: Beta-lactam; Cl: Continuous infusion; ELF: Epithelial lining fluid; IB: Intermittent bolus; Kp: Klebsiella pneumoniae; KPC: Carbapenemase-producing Klebsiella pneumoniae; MIC: Minimum inhibitory concentration; MDR: Multidrug resistant; MRSA: Methicillin-resistant Staphylococcus aureus; PK/PD: Pharmacokinetic/pharmacodynamics; TDM: Therapeutic drug monitoring; VRE: Vancomycin-resistant enterococci.

\section{Author details}

${ }^{1}$ Infectious Diseases Division, Santa Maria Misericordia University Hospital, Udine, Italy. ${ }^{2}$ 4th Department of Internal Medicine, Athens University School of Medicine, Attikon University General Hospital, Athens, Greece. ${ }^{3}$ Université Paris Diderot/Hopital Bichat, Réanimation Medicale et des maladies infectieuses, Paris, France. ${ }^{4}$ UMR 1137, IAME Team 5, Decision SCiences in Infectious Diseases, Control and Care (DeSCID), Inserm/Univ Paris Diderot, Sorbonne Paris Cité, 75018 Paris, France.

Received: 29 February 2016 Accepted: 22 March 2016 Published online: 4 April 2016

\section{References}

1. Holmes AH, Moore LS, Sundsfjord A, Steinbakk M, Regmi S, Karkey A, Guerin PJ, Piddock LJ (2016) Understanding the mechanisms and drivers of antimicrobial resistance. Lancet 387:176-187. doi:10.1016/ S0140-6736(15)00473-0

2. Carlet J (2015) Ten tips on how to win the war against resistance to antibiotics. Intensive Care Med 41:899-901. doi:10.1007/s00134-014-3594-Z

3. Bretonnière $C$, Leone $M$, Milési $C$, Allaouchiche $B$, Armand-Lefevre L, Baldesi O, Bouadma L, Decré D, Figueiredo S, Gauzit R et al (2015) Strategies to reduce curative antibiotic therapy in intensive care units (adult and paediatric). Intensive Care Med 41:1181-1196. doi:10.1007/ s00134-015-3853-7

4. Hranjec T, Rosenberger LH, Swenson B, Metzger R, Flohr TR, Politano AD, Riccio LM, Popovsky KA, Sawyer RG (2012) Aggressive versus conservative initiation of antimicrobial treatment in critically ill surgical patients with suspected intensive-care-unit-acquired infection: a quasi-experimental, before and after observational cohort study. Lancet Infect Dis 12:774780. doi:10.1016/S1473-3099(12)70151-2

5. Garnacho-Montero J, Dimopoulos G, Poulakou G, Akova M, Cisneros JM, De Waele J, Petrosillo N, Seifert H, Timsit JF, Vila J et al (2015) Task force on management and prevention of Acinetobacter baumannii infections in the ICU. Intensive Care Med 41:2057-2075. doi:10.1007/ s00134-015-4079-4

6. Mimoz O, Lucet JC, Kerforne T, Pascal J, Souweine B, Goudet V, Mercat A, Bouadma L, Lasocki S, Alfandari S et al (2015) Skin antisepsis with chlorhexidine-alcohol versus povidone iodine-alcohol, with and without skin scrubbing, for prevention of intravascular-catheter-related infection (CLEAN): an open-label, multicentre, randomised, controlled, two-by-two factorial trial. Lancet 386:2069-2077. doi:10.1016/S0140-6736(15)00244-5

7. Noto MJ, Wheeler AP (2015) Understanding chlorhexidine decolonization strategies. Intensive Care Med 41:1351-1354. doi:10.1007/ s00134-015-3846-6

8. Bassetti M, De Waele JJ, Eggimann P, Garnacho-Montero J, Kahlmeter G, Menichetti F, Nicolau DP, Paiva JA, Tumbarello M, Welte T, Wilcox M, Zahar JR, Poulakou G (2015) Preventive and therapeutic strategies in critically ill patients with highly resistant bacteria. Intensive Care Med 41:776-795. doi:10.1007/s00134-015-3719-z

9. Tumbarello M, Trecarichi EM, De Rosa FG, Giannella M, Giacobbe DR, Bassetti M, Losito AR, Bartoletti M, Del Bono V, Corcione S et al (2015)
Infections caused by KPC-producing Klebsiella pneumoniae: differences in therapy and mortality in a multicentre study. J Antimicrob Chemother 70:2133-2143. doi:10.1093/jac/dkv086

10. De Waele J, Lipman J, Akova M, Bassetti M, Dimopoulos G, Kaukonen M, Koulenti D, Martin C, Montravers P, Rello J et al (2014) Risk factors for target non-attainment during empirical treatment with $\beta$-lactam antibiotics in critically ill patients. Intensive Care Med 40:1340-1351. doi:10.1007/ s00134-014-3403-8

11. Abdul-Aziz MH, Sulaiman H, Mat-Nor MB, Rai V, Wong KK, Hasan MS, Abd Rahman AN, Jamal JA, Wallis SC, Lipman J, Staatz CE et al (2016) Betalactam infusion in severe sepsis (BLISS): a prospective, two-centre, openlabelled randomized controlled trial of continuous versus intermittent beta-lactam infusion in critically ill patients with severe sepsis. Intensive Care Med. doi:10.1007/s00134-015-4188-0

12. Dulhunty JM, Roberts JA, Davis JS, Webb SA, Bellomo R, Gomersall C, Shirwadkar C, Eastwood GM, Myburgh J, Paterson DL (2015) A multicenter randomized trial of continuous versus intermittent $\beta$-lactam infusion in severe sepsis. Am J Respir Crit Care Med 192:1298-1305. doi:10.1164/rccm.201505-08570C

13. Roberts JA, Paul SK, Akova M, Bassetti M, De Waele JJ, Dimopoulos G, Kaukonen KM, Koulenti D, Martin C, Montravers P et al (2014) DALI: defining antibiotic levels in intensive care unit patients: are current $\beta$-lactam antibiotic doses sufficient for critically ill patients? Clin Infect Dis 58:1072-1083. doi:10.1093/cid/ciu027

14. De Waele JJ, Carrette S, Carlier M, Stove V, Boelens J, Claeys G, LerouxRoels I, Hoste E, Depuydt P, Decruyenaere J et al (2014) Therapeutic drug monitoring-based dose optimisation of piperacillin and meropenem: a randomised controlled trial. Intensive Care Med 40:380-387. doi:10.1007/ s00134-013-3187-2

15. de Montmollin E, Bouadma L, Gault N, Mourvillier B, Mariotte E, Chemam S, Massias L, Papy E, Tubach F, Wolff M, Sonneville R (2014) Predictors of insufficient amikacin peak concentration in critically ill patients receiving a $25 \mathrm{mg} / \mathrm{kg}$ total body weight regimen. Intensive Care Med 40:998-1005. doi:10.1007/s00134-014-3276-x

16. Montravers P, Dupont H, Bedos JP, Bret P, Tigecycline Group (2014) Tigecycline use in critically ill patients: a multicentre prospective observational study in the intensive care setting. Intensive Care Med 40:988-997. doi:10.1007/s00134-014-3323-7

17. De Pascale G, Fortuna S, Tumbarello M, Cutuli SL, Vallecoccia M, Spanu T, Bello G, Montini L, Pennisi MA, Navarra P et al (2015) Linezolid plasma and intrapulmonary concentrations in critically ill obese patients with ventilator-associated pneumonia: intermittent vs continuous administration. Intensive Care Med 41:103-110. doi:10.1007/s00134-014-3550-y

18. Tabah A, Cotta MO, Garnacho-Montero J, Schouten J, Roberts JA, Lipman J, Tacey M, Timsit JF, Leone M, Zahar JR et al (2016) A systematic review of the definitions, determinants and clinical outcomes of antimicrobial de-escalation in the intensive care unit. Clin Infect Dis 62(8):1009-17

19. Garnacho-Montero J, Gutiérrez-Pizarraya A, Escoresca-Ortega A, Corcia-Palomo Y, Fernández-Delgado E, Herrera-Melero I, Ortiz-Leyba C, Márquez-Vácaro JA (2014) De-escalation of empirical therapy is associated with lower mortality in patients with severe sepsis and septic shock. Intensive Care Med 40:32-40. doi:10.1007/s00134-013-3077-7

20. Leone $M$, Bechis $C$, Baumstarck K, Lefrant JY, Albanèse J, Jaber S, Lepape A, Constantin JM, Papazian L, Bruder N, Allaouchiche B et al (2014) Deescalation versus continuation of empirical antimicrobial treatment in severe sepsis: a multicenter non-blinded randomized noninferiority trial. Intensive Care Med 40:1399-1408. doi:10.1007/s00134-014-3411-8

21. de Jong E, van Oers JA, Beishuizen A, Vos P, Vermeijden WJ, Haas LE, Loef BG, Dormans T, van Melsen GC, Kluiters YC, Kemperman H et al (2016) Efficacy and safety of procalcitonin guidance in reducing the duration of antibiotic treatment in critically ill patients: a randomised, controlled, open-label trial. Lancet Infect Dis. doi:10.1016/S1473-3099(16)00053-0 